\title{
Analysis of Waveform from Magnetic Printed Perpendicular Hard Disk
}

\author{
N. Sheeda, S. Okami, Y. Sakaguchi, T. Komine and R. Sugita \\ Ibaraki University, 4-12-1 Nakanarusawa-cho, Hitachi, Ibaraki 316-8511, Japan
}

\begin{abstract}
A comparison of waveforms from edge printed and bit printed hard disks at various bit lengths has been discussed by using experimental and simulation studies. Experimental results show that the play-back signal from printed hard disk can be used without difficulty as servo signal when bit length is smaller than $0.4 \mu \mathrm{m}$. Simulation results and frequency spectra obtained by the Fast Fourier Transform show that the waveform from edge printed and bit printed media distorts as the bit length increases.
\end{abstract}

Key words: servo signal, magnetic printing, waveform, bit length

\section{Introduction}

As the application of perpendicular magnetic recording method for hard disk drive (HDD) has been put into practice, a perpendicular magnetic printing that can write the servo signal at high speed, high accuracy and low cost is studied. There are two types of magnetic printing that were investigated in the previous study, which are edge printing (EP) ${ }^{1)}$ and bit printing (BP). However, the previous investigation ${ }^{2), 3)}$ was performed by using master media with bit length that was larger than the actual bit length of commercial hard disk and a comparison of waveforms at various bit lengths has never been discussed yet. In this study, the influence of bit length and printing field on printing characteristics and waveform from edge printed and bit printed media were investigated by using master media with sub micron-scaled patterns and a perpendicular recording hard disk as a slave medium.

\section{Experiments}

Master media that were used in this experiment were made of FeCo film (Co 30 at.\%, $M_{\mathrm{s}}=1900 \mathrm{emu} / \mathrm{cm}^{3}, H_{\mathrm{c}}=$ 70 Oe, $\mu=100$ ) sputtered onto patterned Ni substrate, which was fabricated by EB lithography, sputtering and electroplating techniques ${ }^{4)}$,5). The thickness of the FeCo film is $0.1 \mu \mathrm{m}$. The master media were fabricated into striped pattern consist of about 1000 lines of patterned magnetic films. The ratio of the patterned magnetic film width to the width of the space between patterned magnetic films is nearly equal to $1: 1$ for all bit lengths. The cross-sectional form of the master media is close to the square wave form. In this study, a commercial perpendicular recording hard disk (HD) (saturation field $H_{\mathrm{s}}=10 \mathrm{kOe}$, coercivity $H_{\mathrm{c}}=4.5 \mathrm{kOe}$ ) was used as a slave medium. The thickness of the recording layer and the soft under layer is $20 \mathrm{~nm}$ and $100 \mathrm{~nm}$, respectively. The printed HD was observed by magnetic force microscopy (MFM, PNI product Nano R). Figure 1 shows the process of edge printing (EP) and bit printing (BP). In EP process, printing field $\left(H_{\mathrm{a}}\right)$ is applied in the down track direction of the HD, as shown in Fig. 1(a). In $\mathrm{BP}$ process, $H_{\mathrm{a}}$ is applied in the perpendicular direction to the surface of the HD after the HD was saturated by initial magnetic field in the opposite direction of $H_{\mathrm{a}}$, as shown in Fig. 1(b).
In addition, this analysis was also performed by computer simulation. In order to obtain simulation results that correspond to the experimental results, the master medium in the simulation was set with bit length, $L, 0.1,0.5 \mu \mathrm{m}$, patterned magnetic layer thickness, $\delta_{\mathrm{m}}, 0.1 \mu \mathrm{m}$, track width of infinite, saturation magnetization, $M_{\mathrm{s}}, 1900 \mathrm{emu} / \mathrm{cm}^{3}$, coercivity, $H_{\mathrm{c}}, 0$ Oe, angle dispersion, $\Delta \theta_{50}, 40^{\circ}$ and permeability, $\mu, 100$. In the simulation, first, magnetic field distribution near the master medium was calculated by using the three-dimensional finite element method (3D-FEM) as $H_{\mathrm{a}}$ was applied to the slave medium ${ }^{6}$. Then, the magnetization distribution of the slave medium was computed by the LLG equation with using the magnetic field distribution calculated by the 3D-FEM 7 ). Finally, stray field was calculated from the magnetization distribution of printed HD by using the moment method.
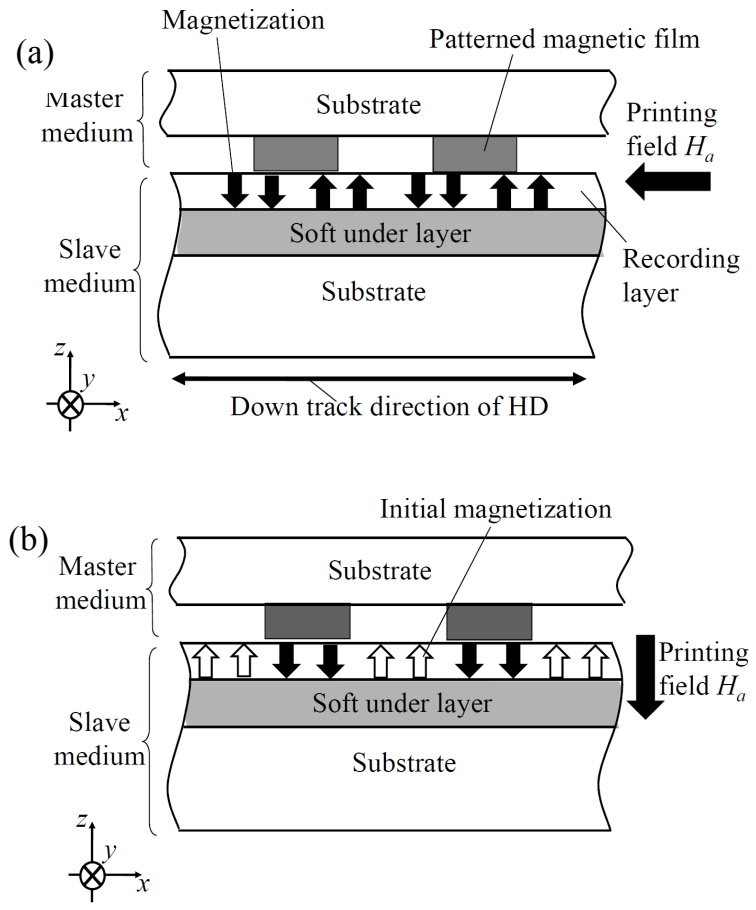

Fig. 1 Schematic diagram of magnetic printing process: (a) edge printing process and (b) bit printing process. 


\section{Results and discussion}

Figure 2 shows the MFM images and MFM output waveforms obtained from edge printed and bit printed medium, respectively with $L=0.1 \mu \mathrm{m}$. The bright part in the MFM image represents the upward facing magnetization while the dark part represents the downward facing magnetization. The printing characteristics were analyzed by calculating the average peak to peak value of the MFM output $V_{\mathrm{p} \text {-p }}$. Table 1 shows the MFM output waveforms obtained from edge printed and bit printed slave media by using master media with $L=0.1,0.25,0.4,0.5 \mu \mathrm{m}$. The $H_{\mathrm{a}}$ for $\mathrm{EP}$ and $\mathrm{BP}$ were set to $8 \mathrm{kOe}$ and $4 \mathrm{kOe}$, respectively, where these are the values for optimum printing characteristics ${ }^{3)}$. From Table 1, it can be seen that the waveforms obtained from edge and bit printed media are nearly sinusoidal when $L$ is smaller than $0.25 \mu \mathrm{m}$, and distort from sine wave when $L=0.4 \mu \mathrm{m}$. In the case of $\mathrm{BP}$ at $L=0.5 \mu \mathrm{m}$, the peak point of the sine wave decreases at the center of the contacted area between slave and master media, which could cause errors during read-back process. Table 2 shows the simulated perpendicular component of magnetization $M_{\mathrm{z}}$ distribution and stray field $H_{\mathrm{z}}$ distribution from the recording layer to compare to the experimental result shown in Table 1 . It shows that the waveform of $H_{z}$ distribution corresponds to the MFM output waveform and distorts from sine wave at longer bit length, similar to the experimental result. The cause of the distortion can be explained by the $M_{\mathrm{z}}$ distribution. In the case of $\mathrm{EP}$ at $L=0.5 \mu \mathrm{m}$, the area between the edges of the patterned magnetic film is almost in demagnetizing state due to the wide gap. In the case of $\mathrm{BP}$ at $L=0.5$ $\mu \mathrm{m}$, the magnetic field generated from the master is weaker at the center of the contacted area compared to the edge area due to the influence of the demagnetizing field. Therefore, the initial magnetization of the slave at the center of the contacted area is hardly reversed by this weak magnetic field. As a result, the $H_{z}$ distribution from the $M_{\mathrm{z}}$ distribution decreases at the center of the contacted area as shown in Table 1 and Table 2. On the other hand, in the case of the non-contacted area, the magnetic field generated from the master is weaker at the center area compared to the edge area due to the decrease of magnetic flux that arises from the magnetic charge of the patterned magnetic film. Therefore, the initial magnetization of the slave at the center of the non-contacted area is easily reversed. As a result, the $M_{\mathrm{z}}$ and $H_{\mathrm{z}}$ distribution decrease at the center of the non-contacted area as shown in Table 2. However, the amount of the wave distortion in the simulation result is slightly larger than the experimental result. The reason for this difference is inferred that the parameters of the calculation model are not sufficiently adapted to the actual medium.

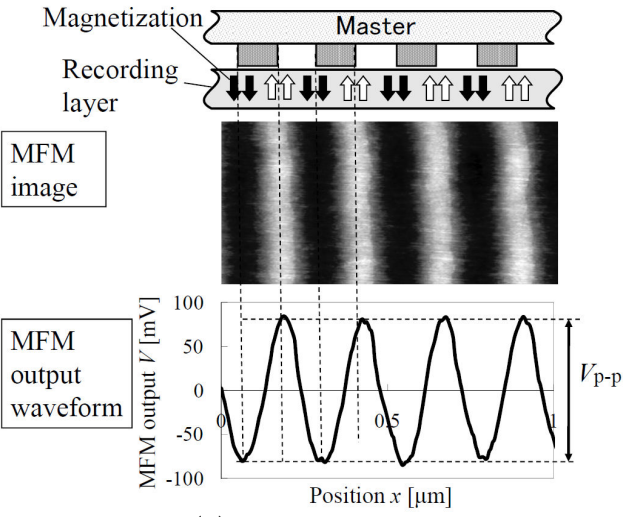

(a) Edge printing

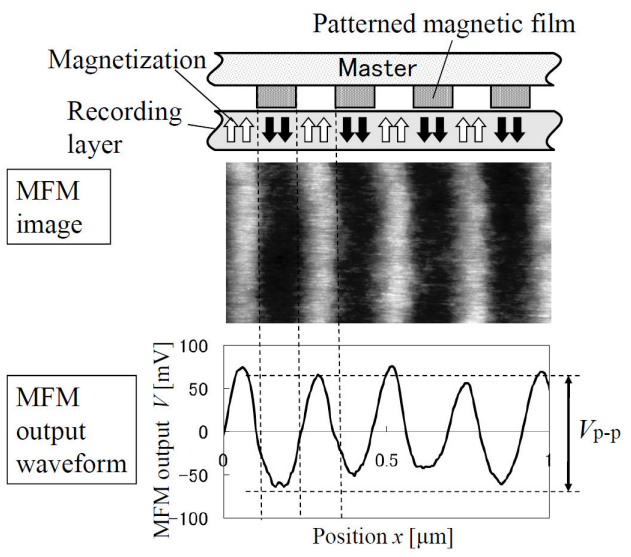

(b) Bit printing

Fig. 2 MFM image and MFM output waveform from (a) edge printed and (b) bit printed medium with $L=0.1$ $\mu \mathrm{m}$.

Table 1 MFM output waveforms from edge printed and bit printed media with various bit lengths.

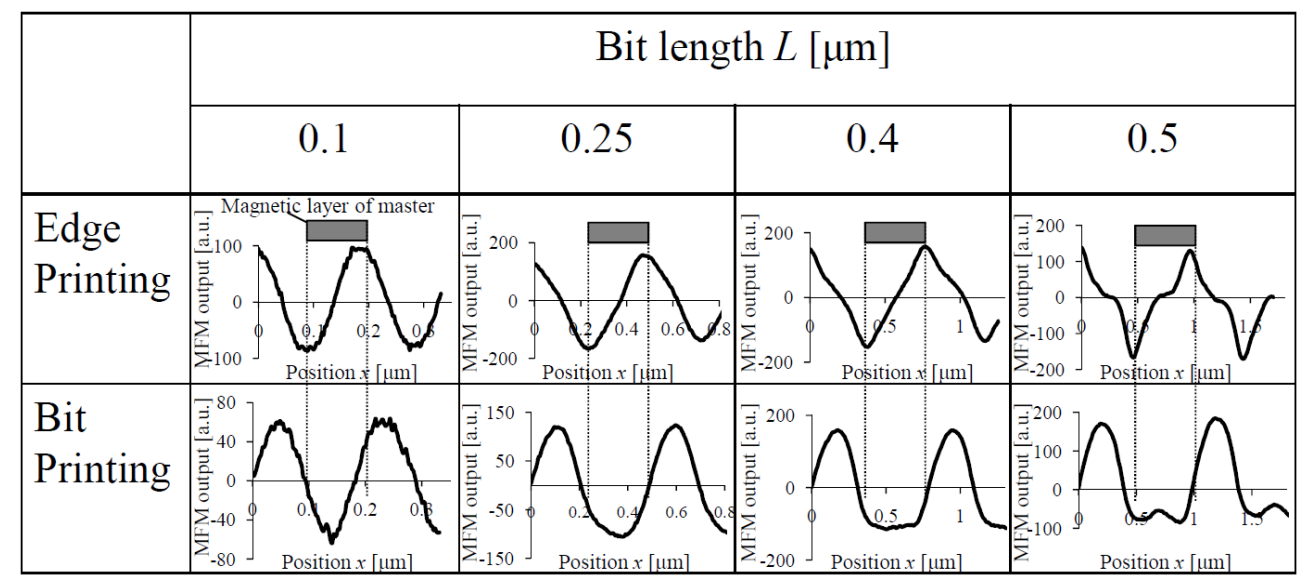


Table 2 Simulated perpendicular component of magnetization distribution $M_{z}$ and stray field distribution $H_{z}$ from edge printed and bit printed media with $L=0.1,0.5 \mu \mathrm{m}$.

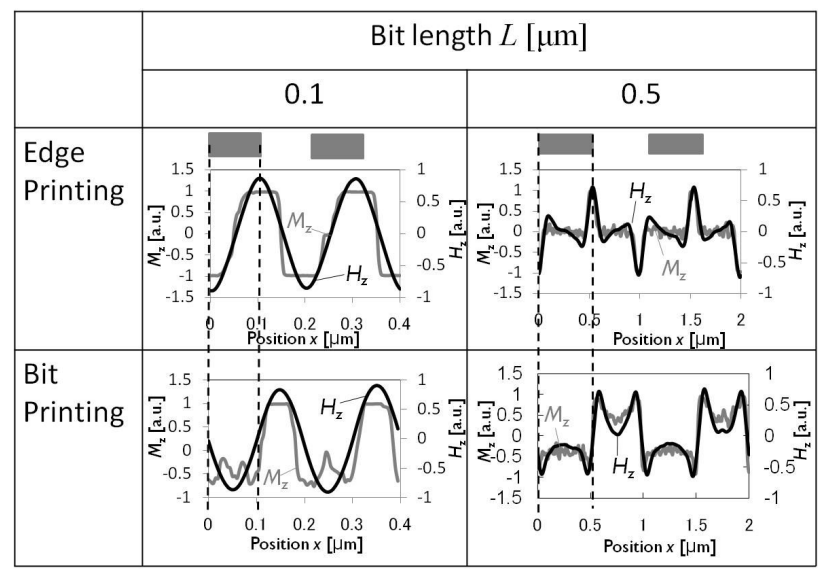

Figure 3 shows the $H_{\mathrm{a}}$ dependence of $V_{\mathrm{p} \text {-p }}$ for EP and $\mathrm{BP}$ by using master medium with $L=0.1 \mu \mathrm{m}$. This result shows that the optimum $H_{\mathrm{a}}$ for $\mathrm{BP}$ is about $4 \mathrm{kOe}$, which is around the coercivity value of the slave medium, while in EP, the magnetization of the recording layer saturates at around $8 \mathrm{kOe}$. This result shows the same tendency as reported in the previous studies ${ }^{3), 6)}$. The bit length dependence of $V_{\mathrm{p} \text {-p }}$ for EP and BP by using the optimum $H_{\mathrm{a}}$ is shown in Fig.4. Printing characteristics in EP deteriorate as the bit length gets shorter because of the increasing demagnetization coefficient. On the other hand, printing characteristics in $\mathrm{BP}$ deteriorate at short bit length due to the decrease of magnetic charge at the surface of the patterned magnetic film as the surface gets smaller. These results correspond with the simulation results in the previous study ${ }^{6}$.

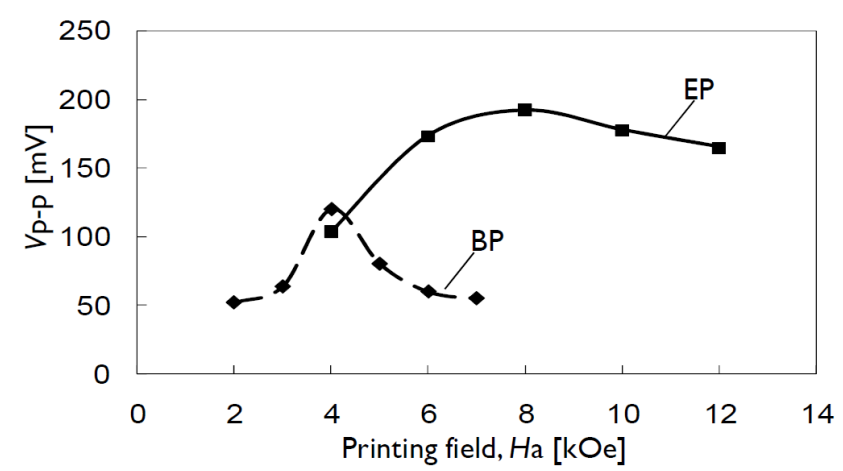

Fig.3 Printing field $H_{\mathrm{a}}$ dependence of $V_{\mathrm{p}-\mathrm{p}}$ for edge printing and bit printing with bit length $L=0.1 \mu \mathrm{m}$.

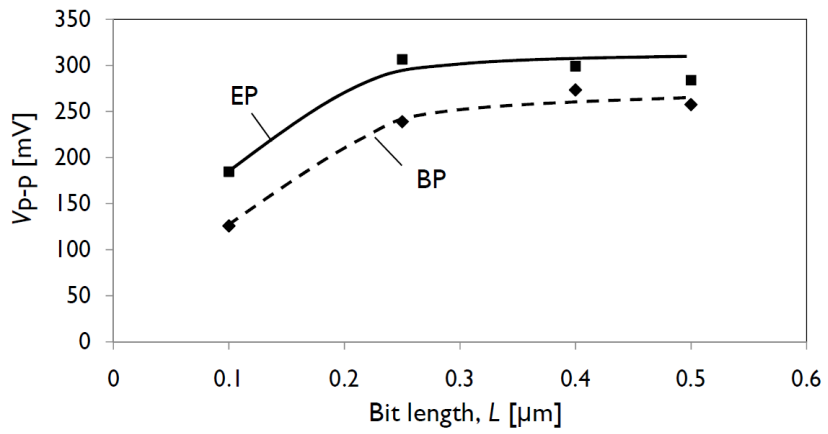

Fig.4 Bit length $L$ dependence of $V_{\mathrm{p} \text {-p }}$ for edge printing with printing field $H_{\mathrm{a}}=8 \mathrm{kOe}$ and bit printing with printing field $H_{\mathrm{a}}=4 \mathrm{kOe}$.

Furthermore, the $L$ dependence of wave distortion was also analyzed by using Fast Fourier Transform (FFT). Figures 5 and 6 show the frequency spectra of the MFM output waveform obtained from edge printed and bit printed media, respectively. In these figures, when $L$ is $0.1 \mu \mathrm{m}$, only the fundamental component exists. However, when $L$ is $0.5 \mu \mathrm{m}$, several harmonic components also exist besides the fundamental component. The $L$ dependence of frequency spectra for $\mathrm{EP}$ and $\mathrm{BP}$ are shown in Figs. 7 and 8, respectively. These figures show that the numbers and the amplitude of harmonic components increase when $L$ increases for both EP and BP.

(a)

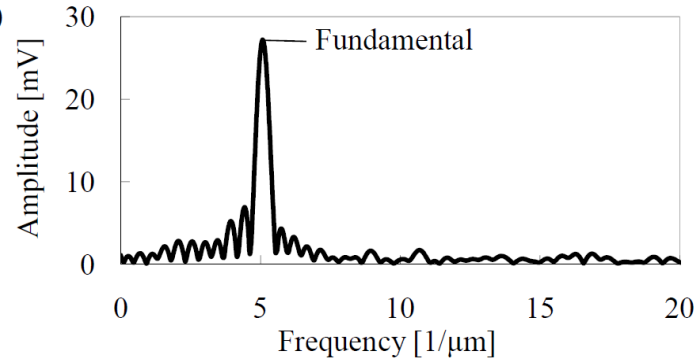

(b)

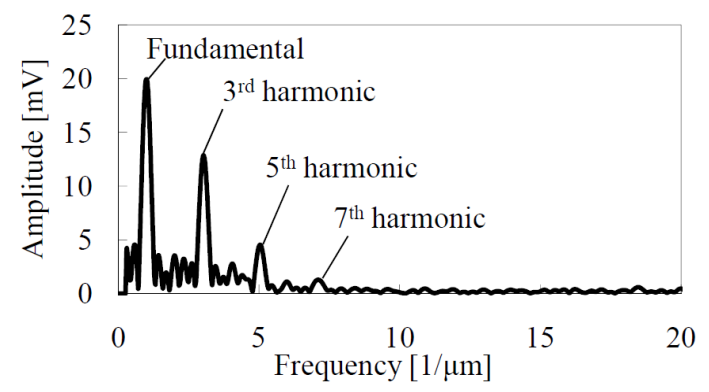

Fig.5 Frequency spectrum of MFM output waveform from edge printed media with (a) bit length $L=0.1 \mu \mathrm{m}$ and (b) bit length $L=0.5 \mu \mathrm{m}$. 
(a)

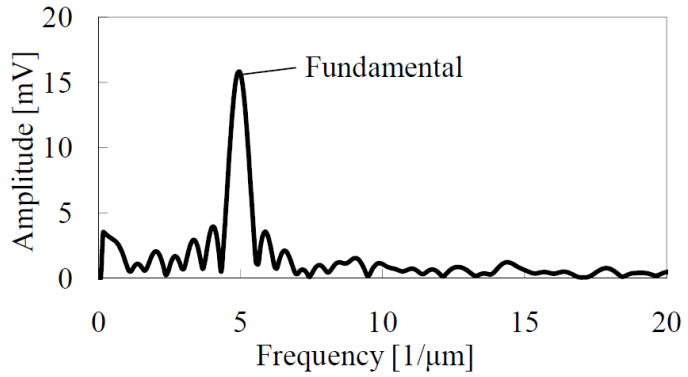

(b)

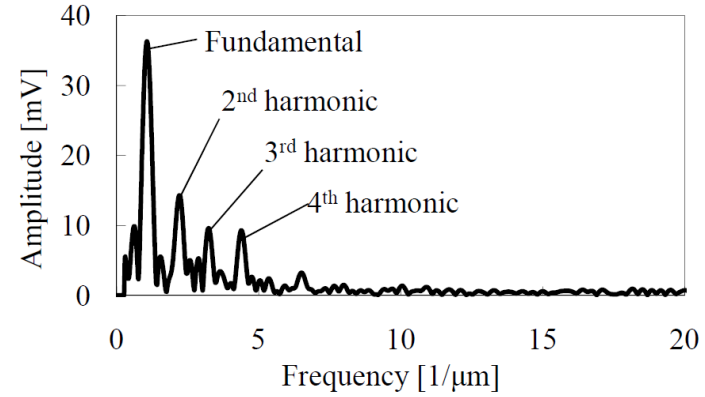

Fig.6 Frequency spectrum of MFM output waveform from bit printed media with (a) bit length $L=0.1 \mu \mathrm{m}$ and (b) bit length $L=0.5 \mu \mathrm{m}$.

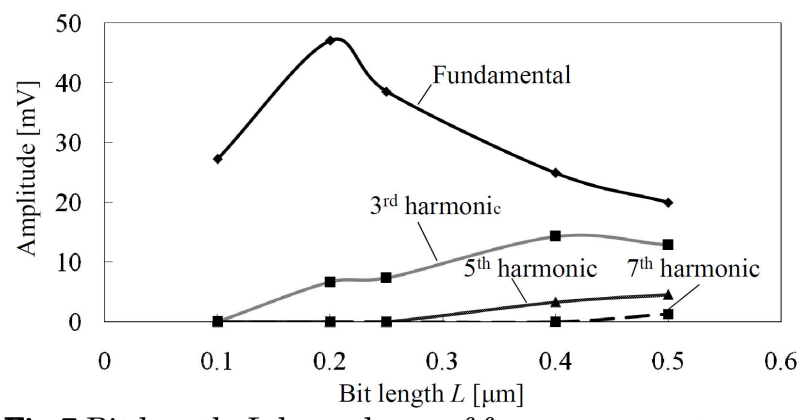

Fig.7 Bit length $L$ dependence of frequency spectrum from edge printed media.

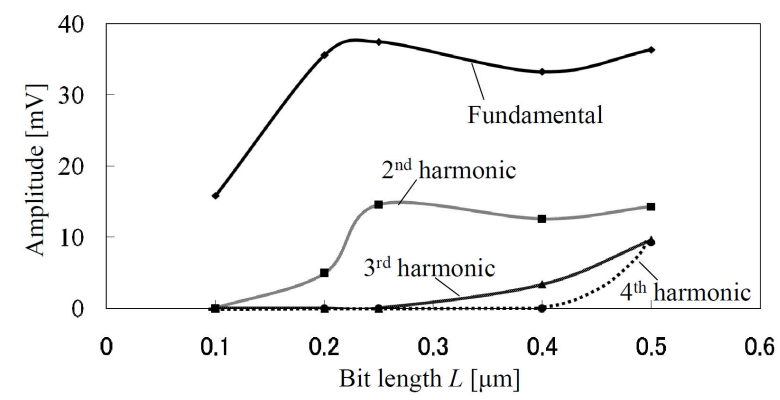

Fig.8 Bit length $L$ dependence of frequency spectrum from bit printed media.

The $L$ dependence of wave distortion in EP and BP is shown in Fig.9. Wave distortion is defined as the following equation:

$$
\text { Wave distortion }=\frac{\sum\left|V_{\text {harmonic }}\right|}{V_{\text {fundamental }}}
$$

Figure 9 shows that the wave distortion increases as $L$ increases in both EP and BP.

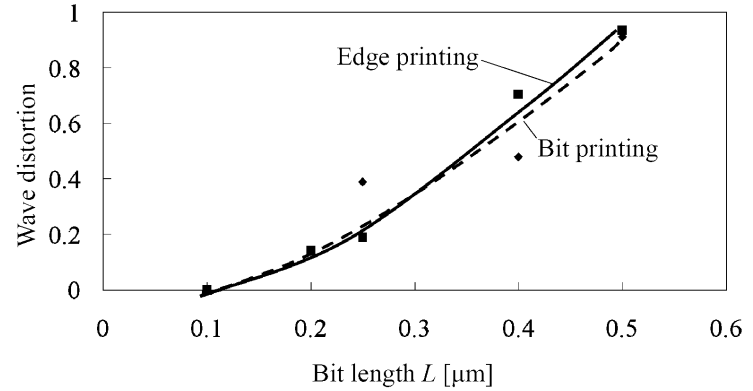

Fig.9 Bit length $L$ dependence of sine wave distortion in edge printing and bit printing.

\section{Conclusion}

A comparison of waveforms from edge printed and bit printed hard disk at various bit lengths has been discussed with using the optimum value of printing field. Printing characteristics for EP and BP are at the best when the printing field is $8 \mathrm{kOe}$ and $4 \mathrm{kOe}$, respectively. Experimental results show that the play-back signal from printed hard disk can be used without difficulty as servo signal when bit length is smaller than $0.4 \mu \mathrm{m}$ for both EP and BP. Frequency spectrum obtained by the Fast Fourier Transform shows that the sine wave from edge printed and bit printed media distorts as the bit length increases. The wave distortion can be explained by the magnetization distribution of the recording layer calculated by LLG. These results show the efficiency of magnetic printing for the present commercial hard disk which has bit length in a range of $50 \mathrm{~nm}$ to $200 \mathrm{~nm}$.

\section{Acknowledgement}

This work was supported in part by NEDO. The authors would like to thank the members of Fuji Film Co., Ltd. for fruitful discussion.

\section{References}

[1] A. Saito, T. Hamada, T. Ishida, Y. Takano, E. Yonezawa, "A Novel Magnetic Printing Technique for Perpendicular Recording Media", IEEE Trans. Magn, 38, 2195-2197, (2002).

[2] N. Sheeda, S. Okami, T. Komine, R. Sugita, "Analysis of Waveform from Perpendicular Magnetic Printed Media", J. Magn. Magn. Mater, 320, 2952-2954, (2008).

[3] S. Okami, R. Ushigome, N. Sheeda, T. Komine, R. Sugita, "Analysis of perpendicular magnetic contact duplication by using metal evaporated tape", J. Magn. Soc. Jpn, 31, no. $3,168-172$, (2007).

[4] A. Morooka, M. Nagao, T. Yasunaga, K. Nishimaki, "Magnetic Recording Performance of "Bit Printing Method" on PMR Media and Its Extendibility to the Higher Areal Density", IEEE Trans. Magn, 44, 3754-3756, (2008).

[5] K. Ichikawa, T. Usa, K. Nishimaki, K. Usuki, "Design oh Ni Mold for Discrete Track Media”, IEEE Trans. Magn, 44, 3450-3453, (2008).

[6] A. Izumi, Y. Nagahama, T. Komine, R. Sugita, T. Muranoi, "Simulation of Magnetic Contact Duplication for Perpendicular Magnetic Recording Media”, J. Magn. Soc. Jpn, 30, 184-187, (2006).

[7]T. Komine, A. Izumi, T. Murata, R. Sugita, "Micromagnetic Simulation of Magnetization States Recorded by Perpendicular Magnetic Duplication", J. Magn. Soc. Jpn, 31, no.3, 173-177, (2007).

Received Oct. 15, 2008; Accepted Mar. 24, 2009 